\title{
Nano ölçekli plakların serbest titreşimi ve tek katmanlı grafen uygulaması
}

\author{
Kadir MERCAN, Çiğdem DEMİR, Ömer CIVALEK* \\ Akdeniz Üniversitesi Mühendislik Fakültesi, Antalya-TÜRKIYE \\ Geliș Tarihi (Recived Date): 26.10.2016 \\ Kabul Tarihi (Accepted Date): 25.01.2017
}

\section{Özet}

Malzeme bilimindeki son gelişmelere paralel olarak nano ölçekli cihazlar pek çok alanda yaygın olarak kullanılmaktadır. Böylece nanoteknoloji günümüzde pek çok bilim dalını ilgilendiren bir araştırma alanı olmuştur. Bu çalışmada, grafen tabakaların sürekli mekanik model ile serbest titreşim hesabı yapılmıştır. Kullanılan mekanik model ince elastik plaktır. Plak için hareket denklemleri elde edilmiş ve hem analitik hem de sayısal olarak çözülmüş̧ür. Analitik çözümde değişkenlere ayırma metodu kullanılmıştır. Sayısal çözüm ise ayrık tekil konvolüsyon yöntemiyle yapılmıştır. Tek katmanlı grafen tabakaların serbest titreşim hesabı plak model için yapılarak sonuçlar tablo halinde verilmiştir. Elde edilen sonuçlara göre, mod sayısı arttıkça doğal ve açısal frekansın büyüdüğ̈̈, modellenen plağın kenar uzunlukları arttıkça da doğal ve açısal frekansin küçüldüğ̈̈ gözlemlenmiştir.

Anahtar kelimeler: Nano-ölçekli modelleme, grafen, plak modeli, titreşim.

\section{Free vibration of nano-scaled plates and application to single- layer graphene}

\begin{abstract}
As parallel to the recent developments in material sciences, nano-scaled devices have widely used in many area. Thus, nanotechnology is being a popular research area which interested many disciplines nowadays. In this study free vibration analysis of graphene sheets has been made via continuous mechanical model. Thin elastic plate

Kadir MERCAN, mercankadir@akdeniz.edu.tr, http://orcid.org/0000-0003-3657-6274

Çiğdem DEMİ, cigdemdemir@akdeniz.edu.tr, http://orcid.org/0000-0002-1890-7220.

* Ömer CIVALEK, civalek@yahoo.com, http://orcid.org/0000-0003-1907-9479
\end{abstract}


model is used in order to make analysis. Equations of motion are obtained for plate model and solved both analytically and numerically. Separation of variables has been used for analytical solution. The numerical solution, however, is obtained via discrete singular convolution method. Free vibration analysis of single-layered graphene sheets is made for plate and results are given in tables. As it can be seen from obtained results, the higher mod number gives higher natural and angular frequencies; on the other hand longer plate dimension gives lower natural and angular frequencies.

Keywords: Nano-scaled modeling, graphene, plate model, vibration.

\section{Giriș}

Günümüzde teknolojik imkânların artmasıyla mikro ve nano boyutlardaki çalışmalara hız verilmiştir. Nanoteknoloji, ülkeler için stratejik bir önem taşımaya başlamış durumdadır. Gelişmiş ülkeler öncelikli alanlarını belirleyip çalışma ve eğitim programlarını geliştirirken, ülkemizde nanoteknoloji araştırmalarının çoğu kuramsal ve bireysel düzeyde kalmıştır. Avrupa Birliğinin 6. Çerçeve Programı sayesinde nanoteknoloji araştırmaları yeniden yapılanma ve ivme kazanmıştır. Aynı zamanda nanoteknoloji, TÜBİTAK tarafından hazırlanan Vizyon 2023 Programında öncelikli alanlardan biri olarak yer almıştır.

Grafen, son zamanlarda oldukça ilgi uyandıran bir malzemedir [1-11]. Grafenler sp ${ }^{2}$ bağ yapısına sahip olan tek tabakalı düzlemsel karbon yapılardır ve üç boyutlu grafitlerin iki boyutlu kopyalarıdır. Mükemmel yapıdaki grafenler, hekzagonal hücrelerden oluşur. Tek duvarlı karbon nanotüpler ise, grafenin silindire yuvarlanmış hali olarak düşünülebilirler. Grafenin gösterdiği önemli özelliklerden biri sıcaklıktan bağımsız 104 $\mathrm{cm}^{2} \mathrm{~V}-1 \mathrm{~s}-1$ değerine ulaşan mobilitesi olup, diğer önemli bir özelliği de Kesirli Kuantum Hall [12] etkisidir.

Membran modeli burkulmaya karşı rijitliği olmayan ince plak modelidir ve yanal güçleri eksenel ve merkezi kesme kuvvetleri ile taşır. Bu nedenle, membran taşıyıcı sistemleri, aşırı incelikleri ve moment taşıma güçlerinin ihmal edilebilir olması nedeniyle gergin kablo ağlarına benzetilebilirler [13-21]. Plak modelinde ise burkulmaya karşı rijitlik vardır ve modellenen tabaka belli bir $h$ kalınlığına sahiptir [12].

Bu çalışmada, grafen bir tabaka sürekli bir model şeklinde çözümlenmiş ve dikdörtgen plak olarak modellenmiştir. Atomik modeller ve deneysel çalışmalar hem pahalı hem de yüksek kapasitede bilgisayar kapasitesi gerektirdiğinden sürekli modeller nanoteknoloji için iyi bir alternatif olmaktadır.

\section{Grafen tabakalar}

Olağanüstü özelliklere sahip bir madde olan ve endüstriyel anlamda alternatif bir yap1 malzemesi olarak kullanılabileceği düşünülen grafen, ince, esnek ve çelikten 100 kat daha güçlü bir maddedir. Grafenin iletkenliği bakırdan daha fazladır ve plastikle \%1 oranında karıştırıldığında plastiği elektrik iletken hale getirebilmektedir. Bunun sonucunda, grafenin elektronik alanındaki silikonun yerine geçebileceği düşünülmektedir [8]. Ayrıca, su 1slahı, petrol sızıntısı temizliği ve hatta çok daha ince 
kondom üretebilmek konusunda da faydası dokunabileceği düşünülmektedir [8].Grafit aslında gündelik hayatta kullanılan çok basit bir eşyada bulunmaktadır. Buna en güzel örneklerin birisi kurşun kalemdir. Kurşun kalemlerdeki kurşunu üretmek için kille karıştırılan grafit, grafen tabakalarının üst üste binmesinden oluşmaktadır. Şekil 1'de tipik bir grafenin bal peteğine benzer dokusu gösterilmiştir. Burada mavi olarak gösterilen küreler karbon atomlarıdır [11-23].

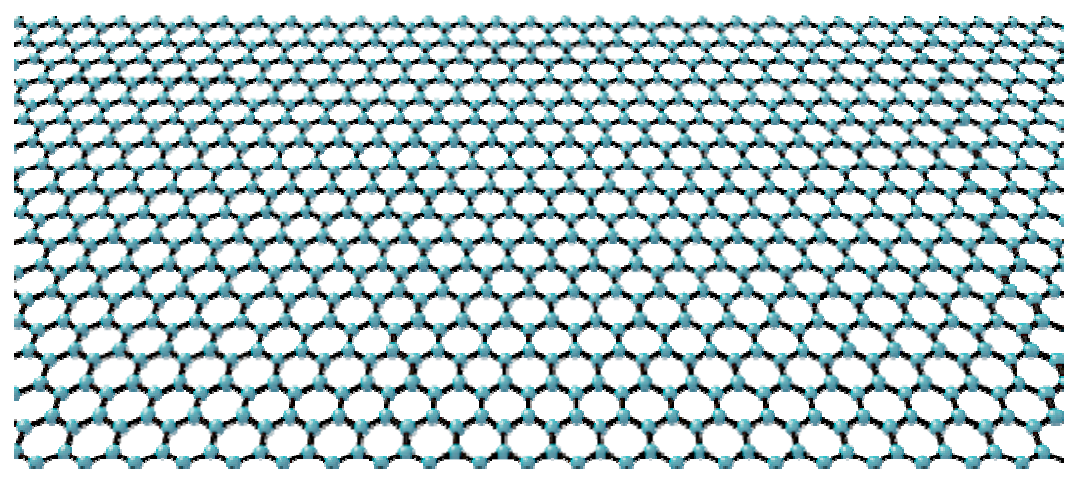

Şekil 1. Tipik bir grafen tabakasının yapısı.

Grafenin rulo haline gelmiş formu olan karbon nanotüpler, günümüzde elektronikten sağlığa birçok alanda kullanılmaktadır [13-21]. Benzer şekilde, grafenin de karbon nanotüpler için öngörülen alanlarda kullanılması mümkündür. Nanotüpler için edinilmiş deneyimlerden yararlanılarak, grafen teknolojisinin önümüzdeki yıllarda geliştirilerek farklı mühendislik alanlarında kullanılması beklenmektedir. Grafenin nanotüplere oranla daha basit olan elde ediliş teknikleri ve bu tekniklerin nanotüplere göre daha kontrol edilebilir olması, grafenin nanotüp teknolojisi üzerine hâkimiyet kurmasını da beraberinde getirebilir [16-23]. Nano ve mikro sistemlerin analizinde atomik benzetim ve moleküler dinamik yaklaşımlar pahalı ve kapsamlı hesaplayıcılar gerektirdiğinden, ayrıca, her durum için deneysel araştırma mümkün olamayacağından sürekli mekanik modeller $[13,24]$ yaygın olarak kullanılmaktadır. Bu modellerin etkinliği pek çok çalışmada ortaya konulmuştur [25-36].

\section{Ayrık tekil konvolüsyon (ATK) yöntemi}

$\mathrm{Bu}$ çalışmada, çözüm için analitik yöntemin yanı sıra sayısal bir yöntemde kullanılmıştır. Bunun için seçilen ayrık tekil konvolüsyon (ATK) yöntemi, ilk olarak Wei[37] tarafından ortaya konulmuştur. $\mathrm{Bu}$ yöntemde, çeşitli fen bilimleri ve mühendislik problemlerinde görülen tekil konvolüsyonlar $(T K)$, Hilbert, Abelve Radon gibi, matematik dönüşümlerinin özel bir sınıfını oluşturmaktadır [38]. Son bir kaç yılda gelişme gösteren matematiğin yeni dalı Wavelet (dalgacık) bu metodun esasını teşkil etmektedir[39]. Ayrık tekil konvolüsyon yöntemi, birçok sayısal yöntemde olduğu gibi, sürekli sisteme ait türev denklemini kerneller kullanarak yaklaşım veya test fonksiyonu olarak ayrıştırır [40-44]. Kernel olarak Shannon kernel, Shannon delta kernel, Dirichlet kernel, de la Vallee kernel vb. kullanılabilir. Eşitlikteki $T$ ve $\eta(t)$ test fonksiyonundaki eleman değerleridir. Tekil konvolüsyon;

$$
F(t)=(T * \eta)(t)=\int_{-\infty}^{\infty} T(t-x) \eta(x) d x
$$


olarak ifade edilebilir [37]. Tekil kernel ise;

$$
T(x)=\delta^{(n)}(x) ; \quad(\mathrm{n}=0,1,2, \ldots) .
$$

Buradaki ayrık kernel, delta türündedir. Kernel eşitliği yüzeysel ve eğrisel interpolasyon için önemlidir ve $n>1$ için türevsel eşitliklerin sayısal çözümünde gereklidir. Yeterli düzgün yaklaşımla, ayrık tekil konvolüsyon yönteminin dikkate alınması son derece etkili olur [39];

$$
F_{\alpha}(t)=\sum_{k} T_{\alpha}\left(t-x_{k}\right) f\left(x_{k}\right)
$$

Yakın geçmişte, bazı yeni kernellerin kullanımı mekanik ve uygulamalı matematik problemlerinin çözümünde önerilmiştir [37-44]. Shannon kernel:

$$
\delta_{\Delta, \sigma}\left(x-x_{k}\right)=\frac{\sin \left[(\pi / \Delta)\left(x-x_{k}\right)\right]}{(\pi / \Delta)\left(x-x_{k}\right)} \exp \left[-\frac{\left(x-x_{k}\right)^{2}}{2 \sigma^{2}}\right]
$$

şeklinde düzenlenmiştir. Burada $\Delta=\pi /(N-1)$ her bir düğüm arası aralık ve $N$ düğüm nokta sayısı, $\sigma$ parametresi Gauss zarfi(Gaussian envelope) genişliğidir ve $\sigma=r h$ şeklinde hesaplanır, $r$ hesaplamanın başında seçilecek bir parametredir. Denklem (4) tekil konvolüsyon kernellerinin (delta türünde) ayrık yaklaşımlar sağlaması için kullanılabilir. Örneğin bir fonksiyon için herhangi bir mertebeden türev şöyle gösterilebilir;

$$
f^{(n)}(x) \approx \sum_{k=-M}^{M} \delta_{\Delta}\left(x-x_{k}\right) f\left(x_{k}\right)
$$

olarak seçilmiştir ve (n) türevin mertebesini göstermektedir.

\section{Grafen tabakanın serbest titreşimleri}

\subsection{Plak teorisi}

Serbest titreşim denklemi, Şekil 2'de verilen uzunluğu $a$, genişliği $b$ ve kalınlığı $h$ olan, homojen ve izotropik dikdörtgen plak için yazılacaktır [12].

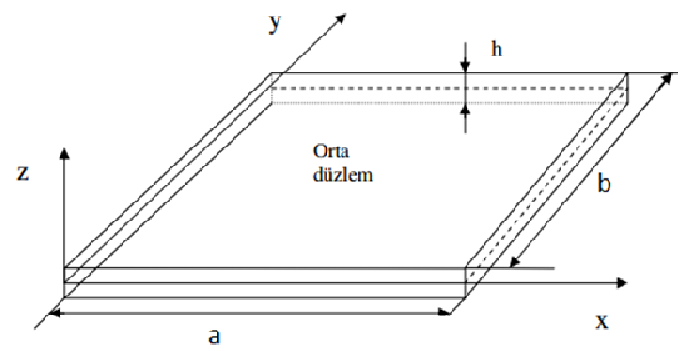

Şekil 2. Dikdörtgen plak 
Plaktaki birim şekil Denklem (6)'da gösterildiği gibi eğrilikler cinsinden hesaplanabilir.

$$
\varepsilon_{x}=z \mathrm{~K}_{x}, \quad \varepsilon_{y}=z \mathrm{~K}_{y}, \quad \gamma_{x y}=2 z \mathrm{~K}_{x y}
$$

Burada $z$, plağın tarafsız eksenden olan uzaklığını, $K_{x}, K_{y}$ ve $K_{x y}$ ise tarafsız eksen eğriliklerini göstermektedir ve aşağıdaki şekilde tanımlanabilir.

$$
\mathrm{K}_{x}=-\frac{\partial^{2} w}{\partial x^{2}}, \quad \mathrm{~K}_{y}=-\frac{\partial^{2} w}{\partial y^{2}}, \quad \mathrm{~K}_{x y}=-\frac{\partial^{2} w}{\partial x \partial y}
$$

Doğrusal elastik malzeme için, plağa ait gerilme bileşenleri eğrilikler cinsinden aşağıdaki şekildedir

$$
\sigma_{x}=\frac{E z}{1-v^{2}}\left(K_{x}+v K_{y}\right), \quad \sigma_{y}=\frac{E z}{1-v^{2}}\left(K_{y}+v K_{x}\right), \quad \tau_{x y}=\frac{E z}{1+v} K_{x y}
$$

Moment-gerilme ilişkisi şu şekilde gösterilebilir;

$$
M_{x}=\int_{-h / 2}^{h / 2} \sigma_{x} z d z, \quad M_{y}=\int_{-h / 2}^{h / 2} \sigma_{y} z d z, \quad M_{x y}=\int_{-h / 2}^{h / 2} \tau_{x y} z d z
$$

Denklem (8), denklem (9)'da yerine yazılırsa moment ile eğrilik arasındaki ilişki aşağıdaki forma dönüşür

$$
\left(\begin{array}{l}
M_{x} \\
M_{y} \\
M_{x y}
\end{array}\right)=D\left[\begin{array}{ccc}
1 & v & 0 \\
v & 1 & 0 \\
0 & 0 & (1-v)
\end{array}\right]\left(\begin{array}{l}
K_{x} \\
K_{y} \\
K_{x y}
\end{array}\right)
$$

Denklem (10)'a denklem (7)'deki ifadeler yazılırsa, moment ifadeleri deplasman cinsinden elde edilmiş olur

$$
\left(\begin{array}{l}
M_{x} \\
M_{y} \\
M_{x y}
\end{array}\right)=-D\left[\begin{array}{ccc}
1 & v & 0 \\
v & 1 & 0 \\
0 & 0 & (1-v)
\end{array}\right]\left(\begin{array}{l}
\frac{\partial^{2} w}{\partial x^{2}} \\
\frac{\partial^{2} w}{\partial y^{2}} \\
\frac{\partial^{2} w}{\partial x \partial y}
\end{array}\right)
$$

Burada, $E$ elastisite modülünü, $v$ poisson oranını, $\rho$ plağın kütle yoğunluğunu (birim alandaki kütle) ifade etmek üzere, $D$ plak eğilme rijitliğini $\left(\frac{E h^{3}}{12\left(1-v^{2}\right)}\right)$ göstermektedir.

Plak üzerinden, kenarları sırasıyla $x$ ve $y$ eksenlerine paralel olan küçük bir dikdörtgen parçası alınacak olursa, plak üzerinde oluşacak iç kuvvetler, eğilme momentleri $M_{x}$ ve 
$M_{y}$, burulma momentleri $M_{x y}$ ve $M_{y x}$, kesme kuvvetleri ise $Q_{x}$ ve $Q_{y}$ şeklindedir (Şekil $3)$.

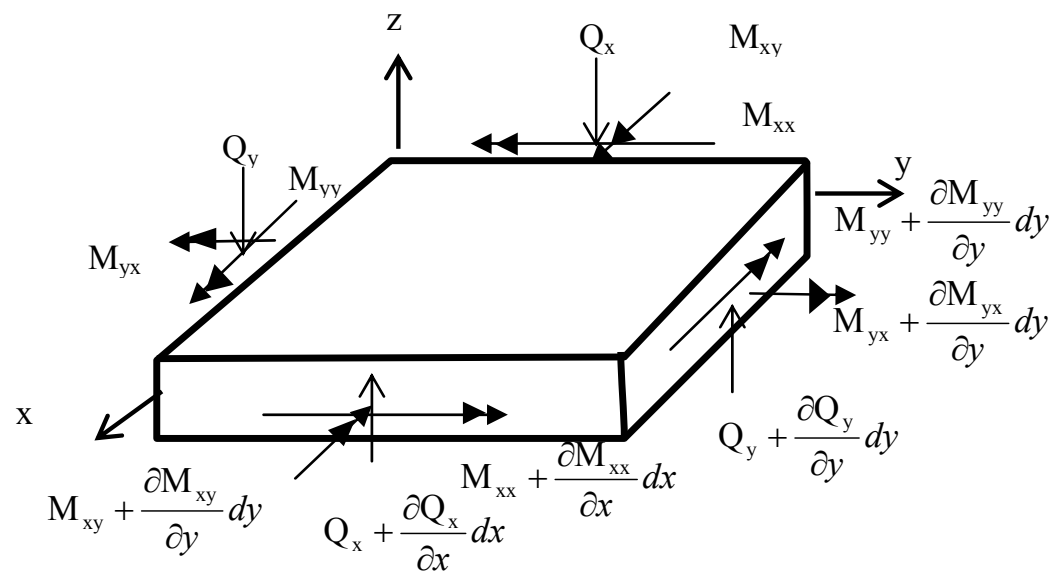

Şekil 3. Plak elemanında oluşan iç kuvvetler

Şekil 3'e göre denge denklemleri yazılacak olursa,

$$
\begin{aligned}
& \frac{\partial Q_{x}}{\partial x}+\frac{\partial Q_{y}}{\partial x}=\rho h \frac{\partial^{2} w}{\partial t^{2}} \\
& Q_{x}=\frac{\partial M_{x}}{\partial x}+\frac{\partial M_{y}}{\partial y} \\
& Q y=\frac{\partial M_{y}}{\partial y}+\frac{\partial M_{x y}}{\partial x}
\end{aligned}
$$

Şeklinde üç adet denklem elde edilmiş olur. Bu durumda denklem (11)'in denklem (12b-c)'de yerlerine yazılmasıyla kesme kuvvetleri şu şekilde yazılabilir;

$$
\begin{aligned}
& Q_{x}=-D \frac{\partial}{\partial x}\left(\frac{\partial^{2} w}{\partial x^{2}}+\frac{\partial^{2} w}{\partial y^{2}}\right) \\
& Q_{y}=-D \frac{\partial}{\partial y}\left(\frac{\partial^{2} w}{\partial x^{2}}+\frac{\partial^{2} w}{\partial y^{2}}\right)
\end{aligned}
$$

son olarak denklem 13(a-b) denklem 12(a)'da yerlerine yazılırsa hareket denklemi deplasmanlara bağlı olarak elde edilmiş olur

$$
D \nabla^{4} w+\rho h \frac{\partial^{2} w}{\partial t^{2}}=0
$$

Burada $\nabla^{4}$ biharmonik operatör olarak isimlendirilir ve aşağıdaki şekilde tanımlanabilir 


$$
\nabla^{4}=\frac{\partial^{4}}{\partial x^{4}}+2 \frac{\partial^{4} w}{\partial x^{2} \partial y^{2}}+\frac{\partial^{4}}{\partial y^{4}}
$$

Problem çözümünden öncelikle olası sınır koşulları belirtilecek olursa, $\mathrm{x}$ doğrultusuna paralel sınır koşulları;

$w=0$ ya da $V_{y}=0$

$$
\frac{\partial w}{\partial y}=0 \text { ya da } M_{y}=0
$$

y doğrultusuna paralel sınır koşulları;

$$
\begin{aligned}
& w=0 \text { ya da } V_{x}=0 \\
& \frac{\partial w}{\partial x}=0 \text { ya da } M_{x}=0
\end{aligned}
$$

x'in sabit bir değeri için denklem (16)'nın farklı mesnet koşulları için ifadesi,

ankastre mesnet;

$w=\frac{\partial w}{\partial y}=0$

basit mesnet;

$w=M_{y}=0$

serbest uç;

$$
M_{y}=V_{y}=0
$$

şeklindedir. Burada $V_{x}$ ve $V_{y}$ Kelvin-Kirchhoff sınır reaksiyonlarıdır ve aşağıdaki şekilde tanımlanabilirler

$$
V_{x}=Q_{x}+\frac{\partial M_{x y}}{\partial y} \operatorname{ve} V_{y}=Q_{y}+\frac{\partial M_{x y}}{\partial x}
$$

Denklem (11) ve denklemler 13(a-b), denklem (19)'daki yerlerine yazılırsa son hali,

$$
V_{x}=-D\left[\frac{\partial^{3} w}{\partial x^{3}}+(2-v) \frac{\partial^{3} w}{\partial x \partial y^{2}}\right] \text { ve } V_{y}=-D\left[\frac{\partial^{3} w}{\partial y^{3}}+(2-v) \frac{\partial^{3} w}{\partial x^{2} \partial y}\right]
$$

olur.

\section{2. Öz dĕger problemi}

Plakların serbest titreşim analizindeki temel sorun uygun sınır koşullarına tabi olan diferansiyel eşitliği çözmektir. Bir plağın titreşim problemini çözmek için denklem (21) 'den yararlanılabilir;

$$
w(x, y, t)=W(x, y) \cos (\omega t+\theta)
$$


Burada, $\theta$ rastgele seçilmiş bir sabiti, $W(x, y)$ ise bilinmeyen bir fonksiyonu göstermektedir. Denklem (21), denklem (14)'te yerine konulursa denklem (22) elde edilir.

$$
\left(\frac{\partial^{4}}{\partial x^{4}}+2 \frac{\partial^{2}}{\partial x^{2}} \frac{\partial^{2}}{\partial y^{2}}+\frac{\partial^{4}}{\partial y^{4}}\right) W(x, y)-\frac{\rho h \omega^{2}}{D} W(x, y)=0
$$

\subsubsection{Dört kenarı basit mesnetli plak modeli}

Navier çözümleri tüm kenarlarından basit mesnetlenmiş bir plak için denklem (23)'de verilen denklem yardımıyla hesaplanabilir;

$$
W(x, y)=A \sin \frac{m \pi x}{a} \sin \frac{n \pi x}{b}
$$

Buradaki $m$ ve $n$ tamsayıları, $A$ ise sıfıra eşit olmayan bir sabit değildir. (23) eşitliğindeki ifade otomatik olarak sınır koşulları özelleştirilmiş olan (22) eşitliğini de sağlanmaktadır. Denklem (22)'de denklem (23) yerine konulduğunda ise şu karakteristik denklem elde edilir;

$$
\pi^{4}\left(\frac{m^{2}}{a^{2}}+\frac{n^{2}}{b^{2}}\right)^{2}-\frac{\rho h \omega^{2}}{D}=0
$$

Yukarıdaki denklemin kökleri plağın doğal frekanslarıdır ve denklem (25)'deki gibi yazılabilir

$$
\omega_{m n}=\pi^{2}\left(\frac{m^{2}}{a^{2}}+\frac{n^{2}}{b^{2}}\right) \sqrt{\frac{D}{\rho h}}, \quad m, n=1,2, \ldots .
$$

Bu işlem Navier çözümü olarak bilinmektedir.

\section{3. İki kenarı basit mesnetli diğer kenarları ankastre ve serbest plak modeli}

Karşılıklı iki kenarı basit mesnetli plaklar için Levy çözümü uygulanmıştır. Plak iki karş1lıklı kenardan basit mesnetlenmiş $(\mathrm{x}=0$ ve $\mathrm{x}=\mathrm{a}$ kenarları) ve diğer iki kenarda $(\mathrm{y}=0$ ve $\mathrm{y}=\mathrm{b})$ rastgele seçilmiş sınır koşullarına sahiptir. Bu rastgele seçilmiş mesnetler, $C$ ve $F$ harfleriyle sırasıyla ankastre ve serbest kenarları göstermektedir.

$$
W(x, y)=Y(y) \sin \frac{m \pi x}{a}
$$

Buradaki $m$ bir tam sayı ve $Y(y)$ belirlenecek bir bilinmeyen fonksiyondur. Denklem(26)' daki sinüs fonksiyonu otomatik olarak sınır koşullarını $\mathrm{x}=0$ ve $\mathrm{x}=\mathrm{b}$ 'de olmasını sağlamaktadır. Levy tipi çözüm olarak bilinen denklem (22)'de yerine yazilırsa,

$$
\frac{d^{4}}{d y^{4}} Y(y)-2 \alpha_{m}^{2} \frac{d^{2}}{d y^{2}} Y(y)+\left(\alpha_{m}^{4}+\gamma^{4}\right) Y(y)=0
$$




$$
\alpha_{m}=\frac{m \pi}{a}
$$

Burada

(i) $0<\gamma<\mathrm{a}_{\mathrm{m}} \quad$ için,

$$
\begin{gathered}
Y(y)=A \cosh \alpha y+B \sinh \alpha y+C \cosh \beta y+D \sinh \beta y \\
\alpha=\sqrt{\alpha_{m}^{2}+\gamma^{2}} \text { ve } \beta=\sqrt{\alpha_{m}^{2}-\gamma^{2}}
\end{gathered}
$$

(ii) $\gamma=\alpha_{m}$ için,

$$
\begin{aligned}
Y(y) & =A \cosh \alpha y+B \sinh \alpha y+C y+D \\
\alpha & =\sqrt{2} \alpha_{m} .
\end{aligned}
$$

(iii) $\gamma \succ a_{m}$ için

$$
\begin{gathered}
Y(y)=A \cosh \alpha y+B \sinh \alpha y+C \cos \beta y+D \sin \beta y \\
\alpha=\sqrt{\alpha_{m}^{2}+\gamma^{2}} \text { ve } \beta=\sqrt{\gamma^{2}+\alpha_{m}^{2}} .
\end{gathered}
$$

Plağın karakteristik eşitliği (29) ile (31) arasındaki eşitlikler sınır koşullarının denklem (22) yerine konularak elde edilir ve plağın doğal frekansları (33) eşitliğinde verildiği gibi elde edilir.

$$
\lambda^{2}=\omega \sqrt{\frac{\rho h}{D}}=\pi^{2}\left(\left(\frac{m}{a}\right)^{2}+\left(\frac{n+(1 / 2)}{b}\right)^{2}\right)
$$

Birleşik mod şekli;

$$
\omega_{m n}=\pi^{2}\left[\left(\frac{m}{a}\right)^{2}+\left(\frac{n+(1 / 2)}{b}\right)^{2}\right] \sqrt{\frac{D}{\rho h}}, \quad f: \frac{\omega}{2 * \pi}
$$

Diğer mesnet koşulları için de benzer şekilde doğal frekans ve birleşik mod denklemleri elde edilebilir.

\section{Sayısal Sonuçlar}

\subsection{Dört kenarı basit mesnetli plak olarak modellenmiş grafen için sonuçlar}

Şekil 4'de, boyutları a ve b olan dikdörtgen plak gösterilmiştir. Plak boyutlarında çeşitli değişikliklere gidilmiş ve farklı boyutlar için farklı sonuçlar elde edilmiştir. Sonuçlar aşağıda Tablo 1'de gösterilmiştir. 


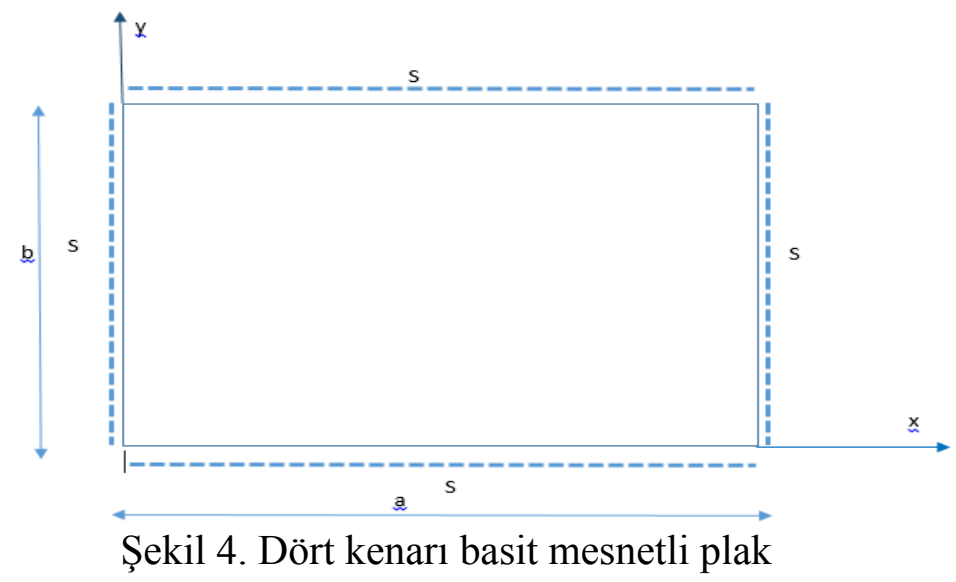

Dikdörtgen plağın açısal frekansı ve açısal frekansa bağlı frekansı şu bağıntıdan elde edilir [12]:

$$
\omega_{m n}=\pi^{2}\left[\left(\frac{m}{a}\right)^{2}+\left(\frac{n}{b}\right)^{2}\right] \sqrt{\frac{D}{\rho h}} \omega_{m n}(x, y)=A_{m n} \sin \frac{m \pi x}{a} \sin \frac{n \pi y}{b}, f: \frac{\omega}{2 * \pi}
$$

Tablo 1.Dört kenarı basit mesnetli dikdörtgen plağın ilk dokuz moddaki açısal frekans ve frekans değerleri $\left(\rho * 10^{-27}=2250 \mathrm{~kg} / \mathrm{nm}^{3}\right)$

\begin{tabular}{|c|c|c|c|c|c|c|c|c|c|c|c|}
\hline \multirow[b]{3}{*}{$\begin{array}{l}\text { Mod } \\
(\mathrm{m}, \mathrm{n})\end{array}$} & \multicolumn{5}{|c|}{$\mathrm{D}=1 \mathrm{nNs} / \mathrm{nm}$} & \multirow{2}{*}{\multicolumn{2}{|c|}{$a=10, b=10$}} & \multirow{2}{*}{\multicolumn{2}{|c|}{$a=10, b=20$}} & \multirow{2}{*}{\multicolumn{2}{|c|}{$a=10, b=30$}} \\
\hline & \multicolumn{2}{|c|}{$a=10, b=10$} & \multicolumn{2}{|c|}{$a=10, b=20$} & $a=10, b=30$ & & & & & & \\
\hline & $\begin{array}{l}\omega^{*} 10^{11} \\
(\mathrm{rad} / \mathrm{s})\end{array}$ & $\begin{array}{c}\mathrm{F} \\
(\mathrm{GHz})\end{array}$ & $\begin{array}{l}\omega^{*} 10^{11} \\
(\mathrm{rad} / \mathrm{s})\end{array}$ & $\begin{array}{c}\mathrm{F} \\
(\mathrm{GHz})\end{array}$ & $\begin{array}{l}\omega^{*} 10^{11} \quad \mathrm{~F} \\
(\mathrm{rad} / \mathrm{s})(\mathrm{GHz})\end{array}$ & $\begin{array}{l}\omega^{*} 10^{11} \\
(\mathrm{rad} / \mathrm{s})\end{array}$ & $\begin{array}{c}\mathrm{F} \\
(\mathrm{GHz})\end{array}$ & $\begin{array}{l}\omega^{*} 10^{11} \\
(\mathrm{rad} / \mathrm{s})\end{array}$ & $\begin{array}{c}\mathrm{F} \\
(\mathrm{GHz})\end{array}$ & $\begin{array}{l}\omega^{*} 10^{11} \\
(\mathrm{rad} / \mathrm{s})\end{array}$ & $\begin{array}{c}\mathrm{F} \\
(\mathrm{GHz})\end{array}$ \\
\hline$(1,1)$ & 1.316 & 20.9 & 0.822 & 13.1 & $0.731 \quad 11.6$ & 1.861 & 29.6 & 1.163 & 18.5 & 1.034 & 16.5 \\
\hline$(1,2)$ & 3.290 & 52.4 & 1.316 & 20.9 & $0.950 \quad 15.1$ & 4.653 & 74.0 & 1.861 & 29.6 & 1.344 & 21.4 \\
\hline$(1,3)$ & 6.580 & 104.7 & 2.138 & 34.0 & 1.31620 .9 & 9.305 & 148.1 & 3.024 & 48.1 & 1.861 & 29.6 \\
\hline$(2,1)$ & 3.290 & 52.4 & 2.796 & 44.5 & $2.705 \quad 43.1$ & 4.653 & 74.0 & 3.955 & 62.9 & 3.825 & 60.9 \\
\hline$(2,2)$ & 5.264 & 83.8 & 3.290 & 52.4 & $2.924 \quad 46.5$ & 7.444 & 118.5 & 4.653 & 74.0 & 4.136 & 65.8 \\
\hline$(2,3)$ & 8.554 & 136.1 & 4.112 & 65.4 & $3.290 \quad 52.4$ & 12.097 & 192.5 & 5.816 & 92.6 & 4.653 & 74.0 \\
\hline$(3,1)$ & 6.580 & 104.7 & 6.086 & 96.9 & $5.995 \quad 95.4$ & 9.305 & 148.1 & 8.607 & 137.0 & 8.478 & 134.9 \\
\hline$(3,2)$ & 8.554 & 136.1 & 6.580 & 104.7 & $6.214 \quad 98.9$ & 12.097 & 192.5 & 9.305 & 148.1 & 8.788 & 139.9 \\
\hline$(3,3)$ & 11.844 & 188.5 & 7.402 & 117.8 & $\begin{array}{lll}6.580 & 104.7\end{array}$ & 16.749 & 266.6 & 10.468 & 166.6 & 9.305 & 148.1 \\
\hline
\end{tabular}

\section{2. İki kenarı basit mesnetli diğer kenarları ankastre ve serbest plak olarak modellenmis grafen için sonuçlar}

Şekil 5'te, boyutları $a$ ve $b$ olan dikdörtgen plak gösterilmiştir. Plak boyutlarında çeşitli değişikliklere gidilmiş ve farklı boyutlar için farklı sonuçlar elde edilmiştir. Sonuçlar Tablo 2'de gösterilmiştir. 


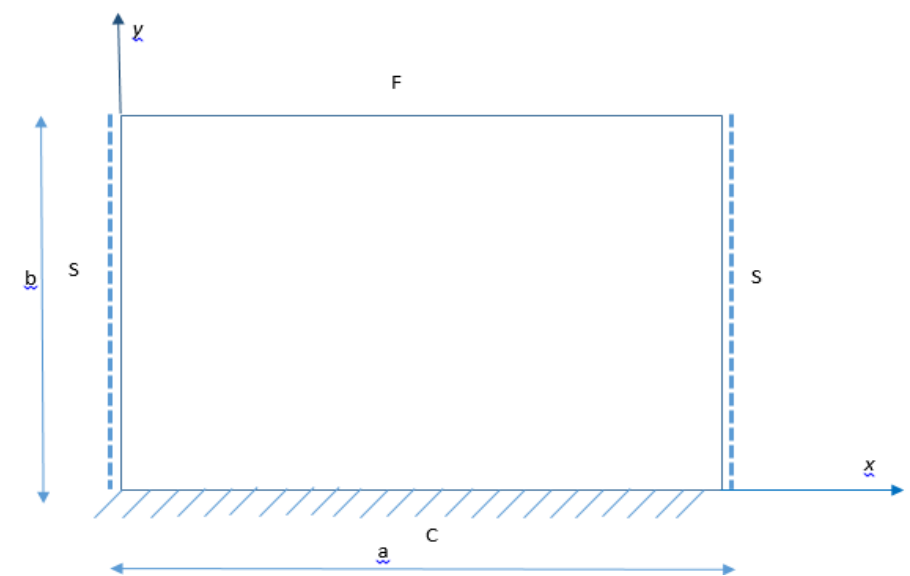

Şekil 5. İki kenarı basit mesnetli diğer kenarları ankastre ve serbest olan plak

Dikdörtgen plağın açısal frekansı ve açısal frekansa bağlı frekansı şu bağıntıdan elde edilir:

$\omega_{m n}=\pi^{2}\left[\left(\frac{m}{a}\right)^{2}+\left(\frac{n+(1 / 2)}{b}\right)^{2}\right] \sqrt{\frac{D}{\rho h}}, \quad f: \frac{\omega}{2 * \pi}$

Tablo 2. İki kenarı basit mesnetli diğer kenarları ankastre ve serbest dikdörtgen plağın ilk dokuz moddaki açısal frekans ve frekans değerleri $\left(\rho * 10^{-27}=2250 \mathrm{~kg} / \mathrm{nm}^{3}\right)$

\begin{tabular}{|c|c|c|c|c|c|c|c|c|c|c|c|c|}
\hline \multirow[b]{3}{*}{$\begin{array}{l}\text { Mod } \\
(m, n)\end{array}$} & \multicolumn{6}{|c|}{$\mathrm{D}=1 \mathrm{nNs} / \mathrm{nm}$} & \multicolumn{6}{|c|}{$\mathrm{D}=2 \mathrm{nNs} / \mathrm{nm}$} \\
\hline & \multicolumn{2}{|c|}{$a=10, b=10$} & \multicolumn{2}{|c|}{$a=10, b=20$} & \multicolumn{2}{|c|}{$a=10, b=30$} & \multicolumn{2}{|c|}{$a=10, b=10$} & \multicolumn{2}{|c|}{$a=10, b=20$} & \multicolumn{2}{|c|}{$\mathrm{a}=10, \mathrm{~b}=30$} \\
\hline & $\begin{array}{l}\omega^{*} 10^{11} \\
(\mathrm{rad} / \mathrm{s})\end{array}$ & $\begin{array}{c}\mathrm{F} \\
(\mathrm{GHz})\end{array}$ & $\begin{array}{l}\omega^{*} 10^{11} \\
(\mathrm{rad} / \mathrm{s})\end{array}$ & $\begin{array}{c}\mathrm{F} \\
(\mathrm{GHz})\end{array}$ & $\begin{array}{l}\omega^{*} 10^{11} \\
(\mathrm{rad} / \mathrm{s})\end{array}$ & $\begin{array}{c}\mathrm{F} \\
(\mathrm{GHz})\end{array}$ & $\begin{array}{l}\omega^{*} 10^{11} \\
(\mathrm{rad} / \mathrm{s})\end{array}$ & $\begin{array}{c}\mathrm{F} \\
(\mathrm{GHz})\end{array}$ & $\begin{array}{l}\omega^{*} 10^{11} \\
(\mathrm{rad} / \mathrm{s})\end{array}$ & $\begin{array}{c}\mathrm{F} \\
(\mathrm{GHz})\end{array}$ & $\begin{array}{l}\omega^{*} 10^{11} \\
(\mathrm{rad} / \mathrm{s})\end{array}$ & $\begin{array}{c}\mathrm{F} \\
(\mathrm{GHz})\end{array}$ \\
\hline$(1,1)$ & 2.138 & 34 & 1.028 & 16.4 & 0.822 & 13.1 & 3.024 & 48.1 & 1.453 & 23.1 & 1.163 & 18.5 \\
\hline$(1,2)$ & 4.770 & 75.9 & 1.686 & 26.8 & 1.115 & 17.7 & 6.746 & 107.4 & 2.384 & 37.9 & 1.576 & 25.1 \\
\hline$(1,3)$ & 8.718 & 138.8 & 2.673 & 42.5 & 1.554 & 24.7 & 12.329 & 196.2 & 3.780 & 60.2 & 2.197 & 35 \\
\hline$(2,1)$ & 4.112 & 65.4 & 3.002 & 47.8 & 2.796 & 44.5 & 5.816 & 92.6 & 4.245 & 67.6 & 3.954 & 62.9 \\
\hline$(2,2)$ & 6.744 & 107.3 & 3.659 & 58.3 & 3.089 & 49.2 & 9.537 & 151.8 & 5.175 & 82.4 & 4.368 & 69.5 \\
\hline$(2,3)$ & 10.692 & 170.2 & 4.646 & 74 & 3.527 & 56.1 & 15.120 & 240.7 & 6.571 & 104.6 & 4.988 & 79.4 \\
\hline$(3,1)$ & 7.402 & 117.8 & 6.291 & 100.1 & 6.086 & 96.9 & 3.024 & 48.1 & 8.898 & 141.6 & 8.607 & 137 \\
\hline$(3,2)$ & 10.034 & 159.7 & 6.949 & 110.6 & 0.822 & 13.1 & 6.746 & 107.4 & 9.828 & 156.4 & 9.020 & 143.6 \\
\hline$(3,3)$ & 13.982 & 222.5 & 1.028 & 16.4 & 1.115 & 17.7 & 12.329 & 196.2 & 1.453 & 23.1 & 1.163 & 18.5 \\
\hline
\end{tabular}

Tablo 1 ve Tablo 2'de sırasıyla dört kenarı basit mesnetli ve iki kenarı basit mesnetli bir kenarı ankastre, bir kenarı serbest dikdörtgen plak için sonuçlar verilmiş olup aralarındaki değişim açıkça gösterilmiştir.

\section{Sonuç}

Bu çalışmada grafen tabakalar bir plak olarak modellenip titreşim analizleri yapılmıştır. Dikdörtgen plaklara ait hareket denklemleri ince plak teorisi için çıkarılarak iki kenarı basit, bir kenarı ankastre, bir kenarı serbest ve dört kenarı basit mesnetliş plak modeller için çözülmüştür. Sonuçlar tablolar halinde sunulmuştur. Tablolardan açıkça görüldüğü gibi plak uzunlukları ve mod sayıları doğal ve açısal frekansa etki etmektedir. Kenar 
uzunluklarının artması frekansları küçültmeye etki ederken mod sayısının artması ise frekansları büyütme yönünde etki ettiği gösterilmiştir. Sürekli mekanik modellerin nanoteknolojide bir alternatif olabileceği vurgulanmıştır.

\section{Teşekkür}

Katkılarından dolayı Akdeniz Üniversitesi BAP birimine teşekkürlerimizi sunarız.

\section{Kaynaklar}

[1] Akgöz, B., Yüksek mertebeden elastisite teorileriyle mikro ve nano yapıların lineer ve lineer olmayan analizleri. Yüksek Lisans Tezi, Akdeniz Üniversitesi, 72 sayfa, (2010).

[2] Ayhan, A., Dünden Bugüne Türkiye'de Bilim-Teknoloji ve Geleceğin Teknolojileri, Beta Basım Yayım Dağıtım, İstanbul, (2002).

[3] Baykara, T., Günay, V. ve Musluoğlu, E., Nanoteknoloji ve nano-malzeme süreçleri. Tübitak MAM, (2010).

[4] Cenger, Y., Nanoteknoloji ve karbon nanoyapılar, Bitirme Tezi, Ankara Üniversitesi, Ankara, (2006).

[5] Çıracı, S., Nanoteknolojide yeni ufuklar, Bilim ve Teknik Dergisi, (2006).

[6] Işık, Ç., Nano ve mikro yapıların lokal olmayan elastisite teorisi ile eğilme ve titreşim hesab1, Yüksek Lisans Tezi, Akdeniz Üniversitesi, Fen Bilimleri Enstitüsü, Antalya, (2011).

[7] Erkoç, Ş., Karbon nano yapılar, Bilim ve Teknik Dergisi, (2001).

[8] Erkoç, Ş., Nanobilim ve Nanoteknoloji, O.D.T.Ü. Bilim ve Toplum Kitapları Dizisi, (2008).

[9] Miyazaki, K. ve Islam, N., Nanotechnology Systems of Innovation - An Analysis of Industry and Academia Research Activities, Technovation, 661675, (2007).

[10] Özer, Y., Nanobilim ve nanoteknoloji: Ülke güvenliği/etkinliği açısından doğru modelin belirlenmesi. Yüksek Lisans Tezi, T.C. Kara Harp Okulu, Ankara, (2008).

[11] Ramsden, J., Nanoteknolojinin Esasları. ÖDTÜ Geliştirme Vakfı Yayıncılık ve İletişim A.Ş. Yayınları, Ankara, (2009).

[12] Leissa, W.A.,Qatu, S.M., Vibration of continuoussystems, TheMcGrow-Hill, (2011).

[13] TÜSİAD, Uluslararası Rekabet Stratejileri: Nanoteknoloji ve Türkiye. Rekabet Stratejileri Dizisi No:11, TÜSİAD-T/2008-11/474, (2008).

[14] Zhang, Y., Tan, Y. W. , Stormer, H. L. , Kim, P., Experimental observation of the quantum Hall effect and Berry's phase in graphene", Nature438: 201-204. doi:10.1038/nature04235, (2005).

[15] Yeğen, F.I., Grafen Şeritler .Yüksek Lisans Tezi, Ankara Üniversitesi, Fen Bilimleri Enstitüsü, Ankara, (2011).

[16] Young, A.F., ve Kim, P., Quantum interference and Klein tunnelling in graphene heterojunctions, NaturePhysics, 5, 222, (2009).

[17] Dede, D., ve Bozkurt, L., Nanoteknoloji, uygulamaları, grafen ve grafen plakaya dikdörtgen membran uygulamasının yapılması. Lisans Bitirme Tezi, Akdeniz Üniversitesi, Antalya, (2013). 
[18] Portugal R., Golebiowski L., ve Frenkel D., Oscillation of membranes using computer algebra, American Journal of Physics, 67, 6, 534-537, (1999).

[19] Novoselov, K. S., Two-dimensional gas of massless Dirac fermions in graphene, Nature438 (7065): 197-200. doi:10.1038/nature04233. PMID 16281030, (2005).

[20] Moğulkoç, A., Grafende kütlesiz dırac fermiyonları gazı, Yüksek Lisans Tezi, Ankara Üniversitesi, Fen Bilimleri Enstitüsü, Ankara, (2008).

[21] Novoselov, K.S. ve Geim, A.K., "The rise of graphene", Nature, 6, 183-191, (2007).

[22] Çıracı, S., Nanoteknolojide yeni ufuklar, Bilim ve Teknik Dergisi, (2005).

[23] Leissa, W.A., ve Qatu, S.M., Vibration of continuous systems, The McGrowHill, (2011).

[24] Luş, H. ve Yerlici, V. 2007., Yapı Dinamiği’ne Giriş., Boğaziçi Üniversitesi Yayınevi, İstanbul, (2007).

[25] Mercan, K., ve Civalek, Ö., DSC method for buckling analysis of boron nitride nanotube (BNNT) surrounded by an elastic matrix, Composite Structures, 143, 300-309, (2016).

[26] Mercan, K., Demir, C., Akgöz, B., ve Civalek, Ö., Coordinate Transformation for Sector and Annular Sector Shaped Graphene Sheets on Silicone Matrix. International Journal of Engineering \& Applied Sciences, 7, 2, 56-73, (2015).

[27] Civalek, Ö., ve Akgöz, B., Static analysis of single walled carbon nanotubes (SWCNT) based on Eringen's nonlocal elasticity theory, International Journal of Engineering and Applied Sciences, 1, 2, 47-56, (2009).

[28] Civalek, Ö., ve Akgöz, B., Vibration analysis of micro-scaled sector shaped graphene surrounded by an elastic matrix, Computational Materials Science, 77, 295-303, (2013).

[29] Demir, Ç., ve Civalek, Ö., Torsional and longitudinal frequency and wave response of microtubules based on the nonlocal continuum and nonlocal discrete models, Applied Mathematical Modelling, 37, 22, 9355-9367, (2013).

[30] Civalek, Ö., ve Demir, Ç., A simple mathematical model of microtubules surrounded by an elastic matrix by nonlocal finite element method, Applied Mathematics and Computations, 289, 335-352, (2016).

[31] Demir, Ç., ve Civalek, Ö., Nonlocal deflection of microtubules under point load, International Journal of Engineering and Applied Sciences, 7, 3, 33-39, (2015).

[32] Civalek, Ö., ve Demir, Ç., Buckling and bending analyses of cantilever carbon nanotubes using the euler-bernoulli beam theory based on non-local continuum model. Asian Journal of Civil Engineering, 12, 5, 651-661, (2011).

[33] http://en.wikipedia.org/wiki/Graphene 2007 (01.05.2016).

[34] Akgöz, B., Civalek, Ö., Shear deformation beam models for functionally graded microbeams with new shear correction factors, Composite Structures, 112, 214-225, (2014).

[35] Akgöz, B., ve Civalek, Ö., A microstructure-dependent sinusoidal plate model based on the strain gradient elasticity theory, Acta Mechanica, 226, 7, 22772294, (2015).

[36] Akgöz, B., ve Civalek, Ö., A new trigonometric beam model for buckling of strain gradient microbeams, International Journal of Mechanical Sciences, 81, 88-94, (2014). 
[37] Wei G.W., A new algorithm for solving some mechanical problems, Comput. Methods, Applied Mechanics and Engineering, 190, 2017 -2030, (2001).

[38] Wei, G.W., Vibration analysis by discrete singular convolution, Journal of Sound and Vibration, 244, 535-553, (2001).

[39] Wei, G.W., Discrete singular convolution for beam analysis, Engineering Structures, 23, 1045-1053, (2001).

[40] Baltacığlu, A.K., Civalek, Ö., Akgöz, B., ve Demir, F., Large deflection analysis of laminated composite plates resting on nonlinear elastic foundations by the method of discrete singular convolution, International Journal of Pressure Vessels and Piping, 88, 8, 290-300, (2011).

[41] Civalek, Ö., The determination of frequencies of laminated conical shells via the discrete singular convolution method, Journal of Mechanics of Materials and Structures, 1, 1, 163-182, (2006).

[42] Civalek, Ö., ve Gürses, M., Free vibration analysis of rotating cylindrical shells using discrete singular convolution technique, International Journal of Pressure Vessels and Piping, 86 ,10, 677-683, (2009).

[43] Civalek, Ö., Fundamental frequency of isotropic and orthotropic rectangular plates with linearly varying thickness by discrete singular convolution method, Applied Mathematical Modelling, 33 , 10, 3825-3835, (2009).

[44] Demir, Ç, Mercan, K., ve Civalek, Ö., Determination of critical buckling loads of isotropic, FGM and laminated truncated conical panel, Composites Part B: Engineering, 94, 1-10, (2016). 